nachweisen. Allerdings fehlt bei den Produktinformationen ein Warnhinweis für Milchallergiker auf diese Bestandteile.

Möglicherweise kommt es in den Laktosepräparaten durch die hohen Laktosekonzentrationen auch zu nicht enzymatischen Glykosylierungen der Milchproteine, wodurch sich deren Konformation ändert. Diese Änderungen können zur Bildung von größeren Glykoproteinkomplexen mit einer erhöhten Allergenität führen. So ergab sich im Intrakutantest eine 10- bis 100fach gesteigerte Reaktivität auf b-Laktoglobulin-Laktose-Konjugate im Vergleich zu nativem b-Laktoglobulin. Eine ungleiche Verteilung dieser hochallergenen Milchproteinkonjugate in den einzelnen Chargen des Advair Diskus ${ }^{\circledR}$ könnte eine Erklärung für die unterschiedliche Reaktivität des kleinen Asthmapatienten sein.

Fazit: Die Inhalation von laktosehaltigen Pulverzubereitungen kann bei
Asthmapatienten mit Kuhmilchallergie anaphylaktische Reaktionen auslösen. Ursache sind geringe Spuren von Milchprotein, die beim Herstellungsprozess der Laktose aus Magermilch nicht vollständig entfernt werden können.

Nowak-Wegrzyn A et al. Contamination of dry powder inhalers for asthma with milk proteins containing lactose. J Allergy Clin Immunol 2004; 113: 558-60

\title{
Asthmatikernasen sind trügerisch
}

\section{Asthmatiker berichten vielfach, unter Haushaltsmitteln und Kosmetika zugesetzten Duftstoffen zu leiden. Gibt es hierfür objektiv messbare Beweise?}

S eit Jahrzehnten schon werden Putzmitteln und auch Kosmetika diverse Duftstoffe zugesetzt und immer wieder stehen diese im Verdacht, die Schleimhäute zu reizen oder gar Allergien auszulösen. Besonders Asthmatiker berichten über vermehrtes Husten, Kurzatmigkeit, trockene oder tränende Augen, verstopfte Nase oder vermehrtes Nasenlaufen bedingt durch Duftstoffe in ihrer täglichen Umgebung.

Eine amerikanische Arbeitsgruppe ging jetzt der Frage nach, inwieweit solche Produkte die oben genannten Symptome tatsächlich hervorrufen und ob sich diese objektiv messen lassen. Dazu wurden 164 Asthmatiker und 217 Kontrollpersonen rekrutiert. Nach eingehenden Voruntersuchungen wurden diese an 2 aufeinander folgenden Tagen in einem $58 \mathrm{~m}^{2}$ großen Raum am ersten Tag einem Wasseraerosol (Kontrolle), am zweiten Tag randomisiert entweder einem Testaerosol (neuentwickelter Sanitätsduftstoff) oder einem gewöhnlichen Raumluft-Deo (Referenzaerosol) ausgesetzt. Die Spraydauer lag bei maximal 16 Sekunden.

Um objektive Daten zu erhalten, wurden bei den Testteilnehmer jeweils vor Versuchsbeginn, nach 5 Minuten und nach 30 Minuten mittels Spirometer der $\mathrm{FEV}_{1}$-Wert gemessen und mittels vergleichender Fotos die Kon- junktiven hinsichtlich einer vermehrten Kapillarfüllung untersucht. Zusätzlich schnaubten sich alle Teilnehmer rechte und linke Nasenhälfte getrennt und gaben ihr subjektives Empfinden hinsichtlich einer verstopften Nase auf einer Skala von eins bis sieben an. Objektiviert wurden diese Daten mittels

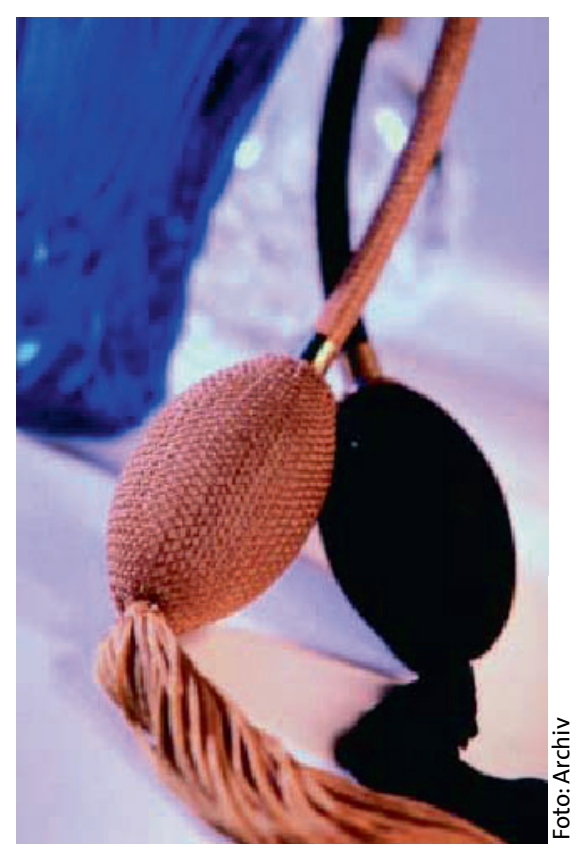

Ist es der Inhalt oder der Anblick eines Parfümzerstäubers, der bei geruchssensiblen Personen einen Reiz auslöst? akustischer Rhinometrie.
Im Ergebnis zeigten sich keine signifikanten Unterschiede der objektiv gemessenen Parameter: Die Kapillarfüllung der Konjunktiven unterschied sich nicht signifikant zwischen den Probanden $(p=0,33)$. Auch gab es keinen signifikanten Unterschied der Reaktion auf die einzelnen Aerosole $(\mathrm{p}=0,36)$. Die erwarteten Veränderungen an den Nasenschleimhäuten blieben aus, die Rhinometrie zeigte keine signifikanten Unterschiede. Ebenso wenig messbare Veränderungen gab es bei der Lungenfunktionsprüfung. Die subjektiv empfundene Luftdurchgängigkeit der Nase war jedoch bei Patienten mit moderatem Asthma sowohl 5 als auch $30 \mathrm{Mi}$ nuten nach Exposition mit dem Referenzaerosol verglichen mit dem Kontrollaerosol signifikant vermindert $(\mathrm{p}=0,032, \mathrm{p}=0,005)$. Auch wenn die objektiv messbaren Werte sich nicht statistisch signifikant veränderten, reagierten die Asthmatiker subjektiv also auf die angebotenen Duftstoffe.

Fazit: Es konnte keine objektiv messbare nasale oder okuläre Irritation durch Duftstoffe bei den getesteten Asthmatikern festgestellt werden, trotzdem fühlten sich manche Probanden subjektiv beeinträchtigt. $\mathrm{Ob}$ der bloße Anblick einer Duftquelle bereits ausreicht, um einen Reiz auszulösen, soll jetzt mit geruchslosen Stoffen überprüft werden.

\section{oj}

Opiekun RE et al. Assessment of ocular and nasal irritation in asthmatics resulting from fragrance exposure. Clin Exp Allergy 2003; 33: 1256-65 